\title{
Cost-effective and fast handoff scheme in Proxy Mobile IPv6 networks with multicasting support
}

\author{
Illkyun $\operatorname{Im}^{\mathrm{a}}$ and Jongpil Jeong ${ }^{\mathrm{b}, *}$ \\ ${ }^{a}$ SAMSUNG SDS Co. Ltd., Principal Engineer Process Technology R\&D Group Samsung SDS \\ Tower(E), Seoul, Korea \\ ${ }^{\mathrm{b}}$ College of Information and Communications Engineering, Sungkyunkwan Univeristy, Suwon-si, Korea
}

\begin{abstract}
With recent advancements in wireless communication technologies, mobile multicasting is becoming important, in an effort to use network resources more efficiently. In the past, when various mobile IP-based multicast techniques were proposed, the focus was on the costs needed for network delivery to provide multicast services, as well as on minimizing the multicast handover delay. However, it is fundamentally difficult to resolve the problems of handover delay and tunnel convergence for techniques using MIPv6 (Mobile IPv6), a host-based mobility management protocol. To resolve these problems, the networkbased mobility management protocol PMIPv6 (Proxy Mobile IPv6) was standardized. Although performance is improved in PMIPv6 over MIPv6, it still suffers from problems of handover delay and tunnel convergence. To overcome these limitations, a technique called LFH (Low-cost and Fast Handoff) is proposed in this paper, for fast and low-cost mobility management with multicasting support in PMIPv6 networks. To reduce the interactions between the complex multicast routing protocol and multicast messages, a simplified proxy method called MLD (Multicast Listener Discovery) is implemented and modified. Furthermore, a TCR (Tunnel Combination and Reconstruction) algorithm was used in the multicast handover procedure within the LMA (Local Mobility Anchor) domain, as well as in the multicast handover procedure between domains, in order to overcome the problem of tunnel convergence. It was found that, compared to other types of multicast techniques, LFH reduces multicast delay, and requires lower cost.
\end{abstract}

Keywords: PMIPv6, multicast, MLD, low-cost handoff, fast handoff

\section{Introduction}

Scores of mobile multicast technologies have so far been suggested and utilized, as they send out data in an efficient way to multiple listeners. Considered instrumental for real-time multimedia services, mobile multicast technology, often times referred to as Multicast Listener Discovery (MLD), comprises a MLD router and MLD hosts, both of which are referred to in administering 'Dynamic Membership' of the IPv6-form address of listeners, allowed a router for each [1]. Due to the difficulties in multicasting to mobile devices users, some new multicast technologies, with higher capability and performance, have

\footnotetext{
${ }^{*}$ Corresponding author: Jongpil Jeong, College of Information and Communications Engineering, Sungkyunkwan Univeristy, 2066, Seobu-ro, Jangan-gu, Suwon-si, Gyeonggi-do, 440-746, Korea. E-mail: jpjeong@ skku.edu.
} 


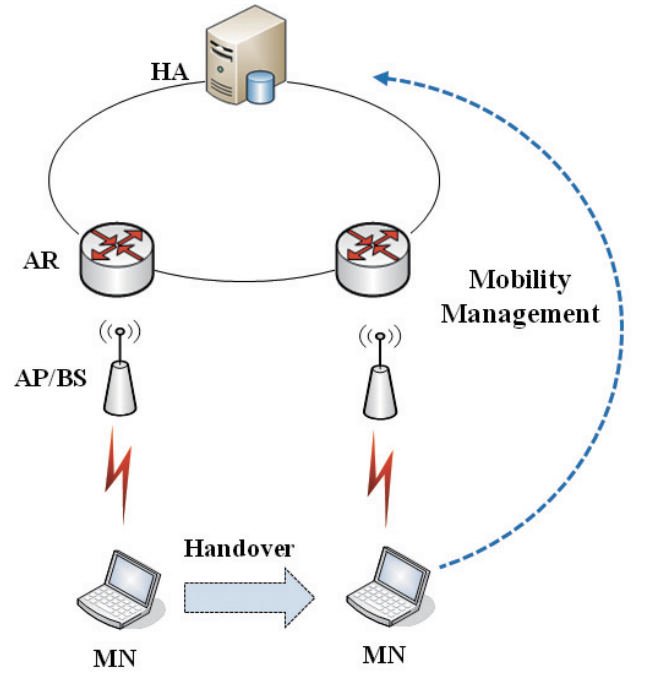

Host-based MIPv6

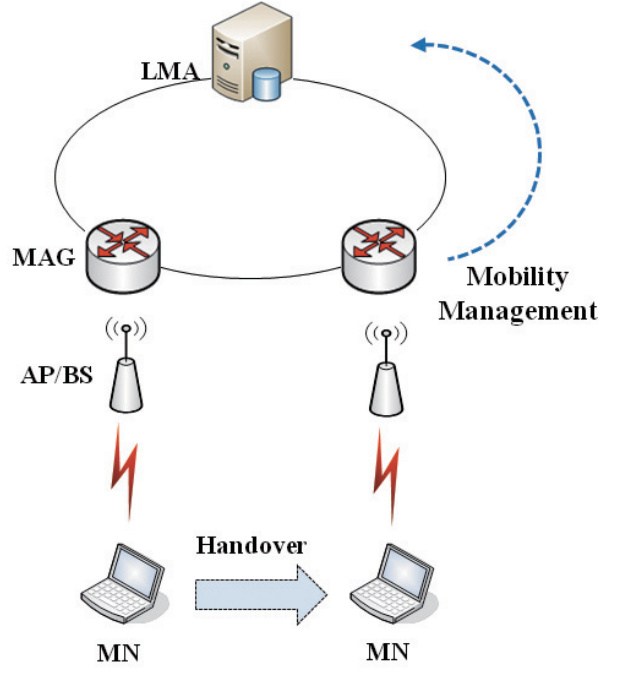

Network-based MIPv6

Fig. 1. Comparison of MIPv6 and PMIPv6.

been introduced, and are becoming popular. These are based on, but not limited to, Mobile IPv6 [2] (MIPv6), the host-based mobility management method, as well as Bi-directional tunneling and Mobile Multicast Protocol) [3], based on 'Remote Subscription', Range-based Mobile Multicast (RBMoM) [4], and Multicast by Multicast Agent protocol (MMA) [5]. However, with improved handover latency and packet loss, they have not been able to go beyond their bounds, of being host-based methods.

The paper thereby suggests PMIPv6 [8], another mobility management standardized method, based on Network-based Localized Mobility Management Working Group (IETF NETLMM WG). As opposed to host-based MIPv6, however, PMIPv6 incorporates network-based methods, where each network entity is entitled to signaling [9]. Note that MN is not under any circumstances incorporated for mobility management, which is supported without any relevant protocol stack, which improves signaling overhead. Still bearing handover latency and tunnel convergence, PMIPv6 incorporates LMA, multicasting data via tunneling, which causes tunnel convergence with inefficiency. While the multicast algorithm of mobile multicast is what data transfer/electricity efficiency is all about, PMIPv6 offers limited support to the intra-domain mobility of MN [10].

The paper also suggests MLD proxying [25] and PMIPv6-based LFH for better cost-efficiency and performance, less overhead, and decent multicasting procedure, as well as being able to support interdomain mobility. Among others, Proxy Binding significantly reduces transferal loss and improves tunnel convergence, by reflecting the TCR algorithm into LFH. Bi-directional tunneling, mobile subscription [11], Multicast Fast Handover (MFH) [12], and Light-weight Mobile Multicast (LMM) [13] have all been incorporated, to prove numerical modeling and performance testing of LFH efficiency and continuity. Note that LFH, as depicted in Figs 10 and 15, features highly-efficient cost of signaling and packets sent, for both inter and intra-domain.

Covering the above contents, the paper comprises PMIPv6 Mobile Multicast in Chapter 2, Intradomain/Inter-domain LFH in Chapter 3, Comparative Advantage of LFH for Multicast Latency and Cost in Chapter 4, and Conclusion in Chapter 5. 


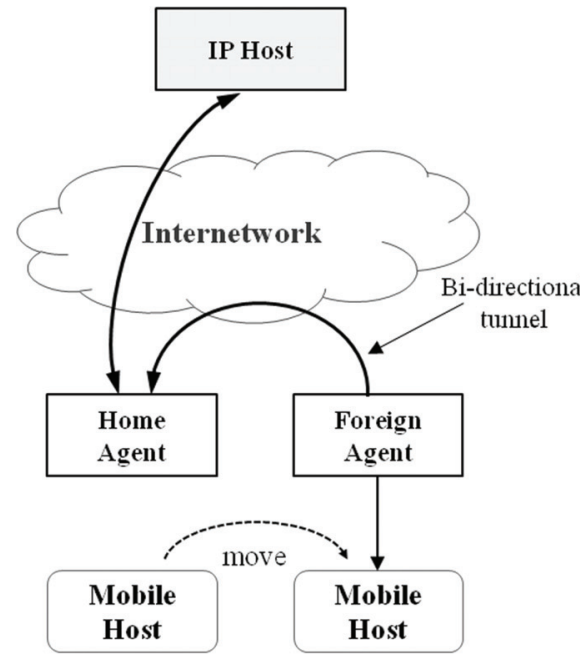

(a)

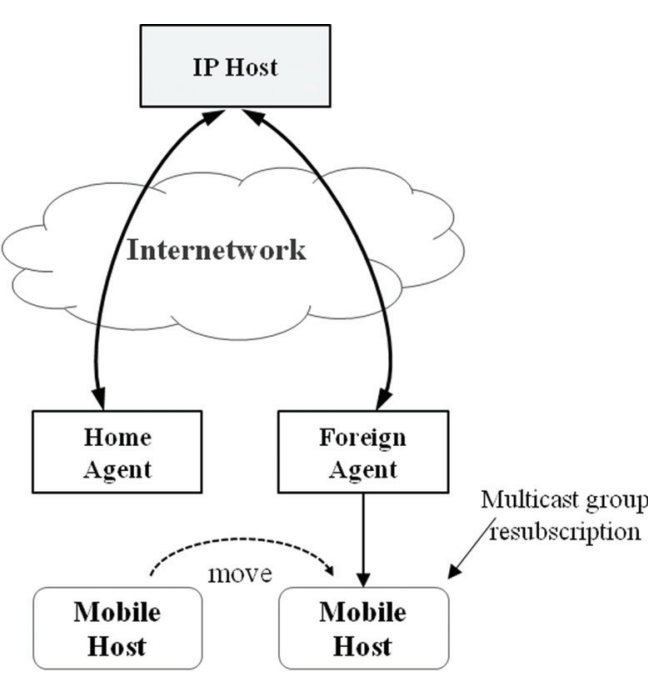

(b)

Fig. 2. (a) Bi-directional tunneling, and (b) Remote subscription.

\section{Related work}

\subsection{Proxy Mobile IPv6}

PMIPv6, as opposed to host-based MIPv6 and HMIPv6, where MNs need to be modified, is a networkbased mobility management protocol, as depicted in Fig. 1. Each network takes part in the mobility management of MN, as opposed to MIPv6, where each MN manages its own mobility. PMIPv6 needs two network entities as Mobility Access Gateway (MAG) and Local Mobility Anchor (LMA). They are responsible for the mobility of MN and administer the reachable intra-domain state of MN network.

\subsection{Expansion of group membership management protocol}

Figure 2 shows the bi-directional tunneling and remote subscription, among other aspects, as suggested by IETF [11].

Note that in bi-directional tunneling, packets travel between Home Agent (HA) and host to feature less latent multicast. This, however, is known to incorporate tunnel convergence, when the multicast data is sent out to multiple unicast tunnels. To improve this, as well as the data transferal route and overlap, the paper suggests remote subscription, transferring of data packets, subscribing to a multicast group, upon the mobility of the host; and not taking a shortcut and packet overlap by way of HA. Remote subscription, however, still incorporates overhead and inefficiency in cost, as 'Multicast Tree' is often reorganized.

Jelger and Noel [14], in an effort to improve this, have suggested a newer multicast, a combination of the bi-directional tunneling and remote subscription, with the use of MLD-proxy-capable HA, where by means of a newer type of MLD messaging, the listeners maintain the 'multicast' state of MN, preventing HA from being multicast. MN, for inter-network mobility, sends out bi-directional tunneling, and turns to the remote subscription method. Upon reception of 'Native Multicast Packets' out of the current access router, MN maintains a 'multicast' state, halting multicasting to HA by sending the relevant message. This, however, incorporates the problem of synchronization [26], of not working properly during the 


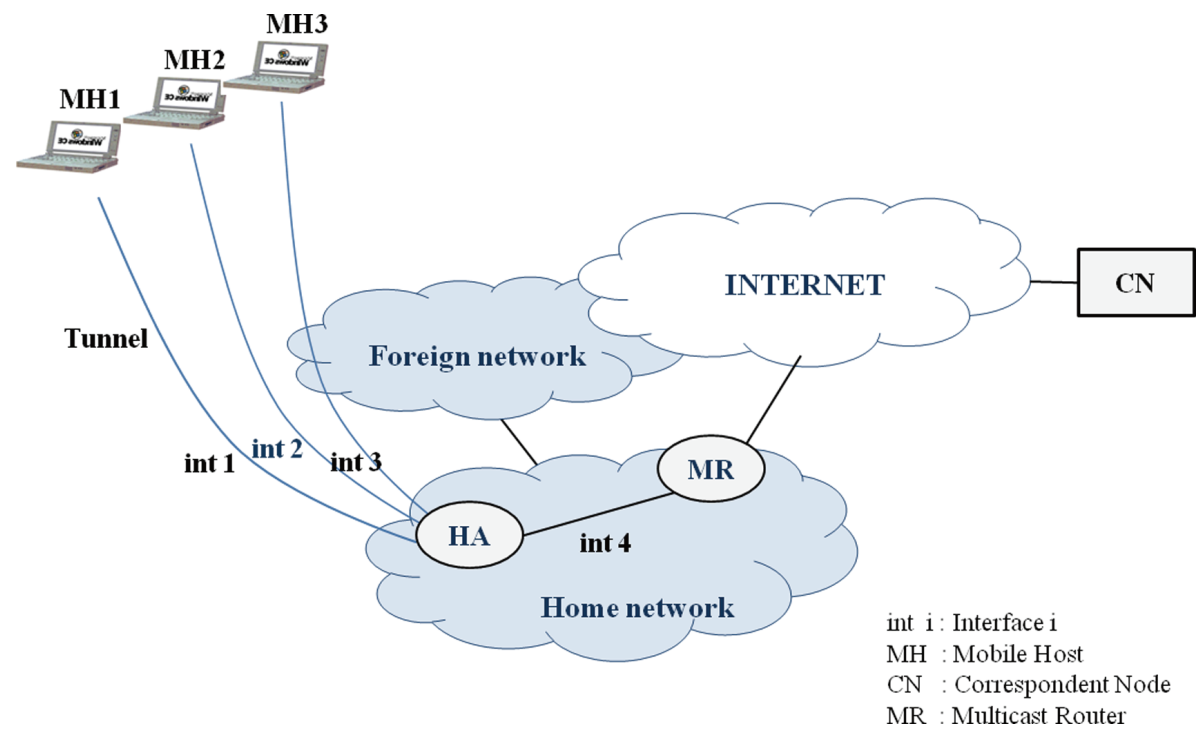

Fig. 3. MLD Multicasting.

course of handover. To improve this, Romdhani [15] has suggested an adaptive multicast membership administration scheme, where the non-multicast HA plays MLD, sending out MLD messages between the multicast router and mobile devices, for multiple mobile devices. This modifies the query message interval, by halting querying upon reception of multicast packet, and thereby saving energy cost for the multicast router and mobile devices. Deering [1] has suggested another method that incorporates MLD for multicast group membership reference, which is demonstrated in Fig. 3. MLD refers to the IPv6 multicast router for membership information to hand over to the multicast routing protocol.

\subsection{Extension of mobility support}

Xia and Sarikaya [12] have suggested Multicast Fast Handover (MFH), on the basis of Fast Handover of Mobile IPv6 (FMIPv6), incorporating messages of Fast Binding Update (FBU) and Handover Initiate (HI), through which PAR sends out group information with efficiency. MFH, however, has bearing latency, later resolved by Leoleis [16] by means of 'conditional tunneling', together with a 'buffering method', to support multicast-handover while improving tunnel convergence. Kwon [17] has suggested another multicast method in which ICMP records the said group information on the header, to prevent tunnel convergence, by setting a multicast tunnel between PAR and NAR. Schmidt and Waehlisch [18] have suggested a near-perfect HMIPv6 hand-off, sending out packets via an anchor point, which Zhang [19] has proposed 'Dynamic Multicast Agent', improving the dynamic hand-off frequency via multicast agent. As opposed to the general mobile multicast methods presuming 'multicastable' AR (subject to HA or IP-multicast), causing inefficient energy consumption of mobile devices and processing overhead, MIPv6 incorporates HA multicasting to the entire IPv6 addresses and Proxy MLD working together with kernel multicast forwarding, which is much more complex, but able to support inter-domain multicast. Proxy MLD is to be performed by MLD messages traded between HAA and $\mathrm{MN}$, involving operational overhead at the home network, especially at HA, by multiple MNs. Yokota [20] has suggested an improved FMIPv6 incorporating less packet loss and latency, by precisely detecting MN to set up a bi-directional tunnel through which packets travel, between pMAG and nMAG, 


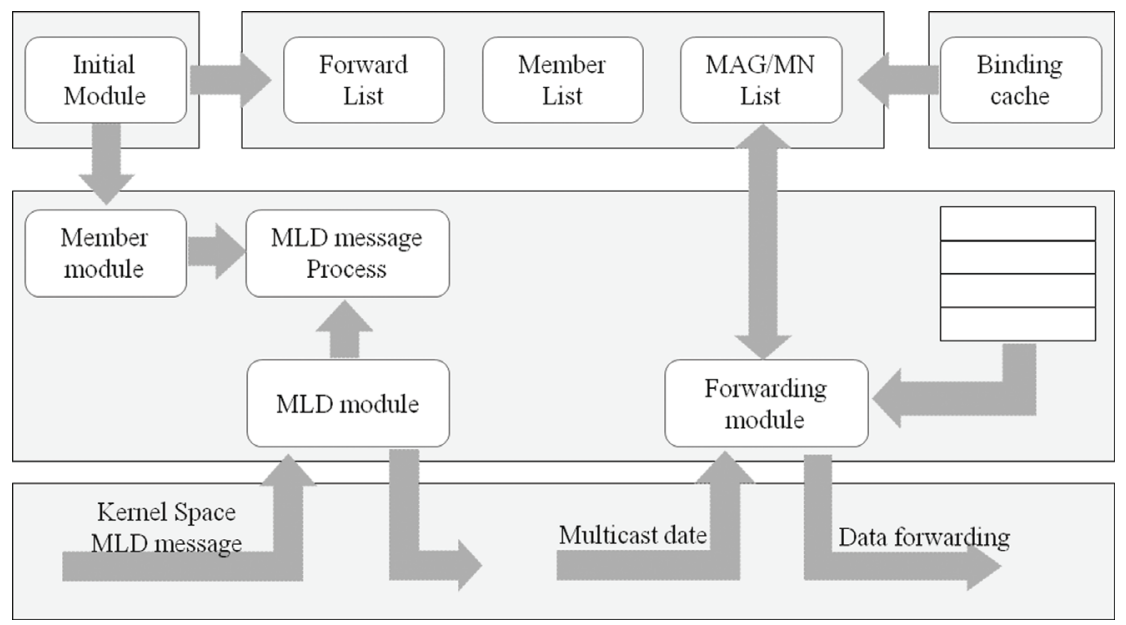

Fig. 4. Streamlined MLD proxying.

after trading $\mathrm{HI}$ and Hack. nMAG, upon recognition of MN access, sends out PBU to LMA with less latency. Lee [21] has suggested a handover method without global mobility of $\mathrm{MN}$, in the way in which post-handover packets track down MN. This, however, incorporates cost inefficiency, as the multiple Local Mobility Domains (LMD) passes MN and CN maintaining a session. Liebsc [22] has suggested an optimized-route algorithm between two MNs, intra-LMD of PMIPv6. This, while supporting networkbased mobility management, does not however take the distant LMD into account. The fundamental multicast method of PMIPv6 [23] trades MLD for membership, after the binding procedure [24]. Upon mobility of MN to another MAG, a binding procedure, as done for access to pMAG, is to be made, incorporating latency. MULTIMOB WG has been suggested, to prevent latency, offering a faster multicast handover [6]. Note that another method of Fast PMIPv6 (FPMIPv6) $[9,10]$ improves handover capability of PMIPv6. FPMIPv6, like PMIPv6 and without any functional addition, still has room, however, for MAG and LMA to develop further. Like PMIPv6, the bi-directional tunnel between pMAG and nMAG is projected to reduce packet loss.

\section{Proposed LFH scheme}

\subsection{LFH: Fundamental structure}

LMA transfers multicast packets, as well as LMD featuring streamlined LMD proxy, between multicast router and listener [25]. This MLD proxy obtains and maintains the multicast group information learnt, while the router queries internet group management protocol to the local network. Upon receiving queries, the host elements are to respond by sending out a report. MLD proxy comprises the upper layer interface, linked to the router, and lower layer interface associated with the subscriber of multicast. Note that any information of lower layer interface is to be recorded by MLD proxy. Note further that LMA, for the MLD proxy, is deemed independent of the routing protocol of the fixed network. As MN, upon mobility, is unable to be halted by MLD query, LFH streamlines MLD proxy to reduce operational overhead, thereby sending out limited message and state of access to the said MLD proxy. LFH, upon execution of MLD proxy streamlining, is to compose the interface, linked to the upper layer multicast 
$\begin{array}{llllllllllllllllllllllllllllllll}0 & 1 & 2 & 3 & 4 & 5 & 6 & 7 & 8 & 9 & 0 & 1 & 2 & 3 & 4 & 5 & 6 & 7 & 8 & 9 & 0 & 1 & 2 & 3 & 4 & 5 & 6 & 7 & 8 & 9 & 0 & 1\end{array}$

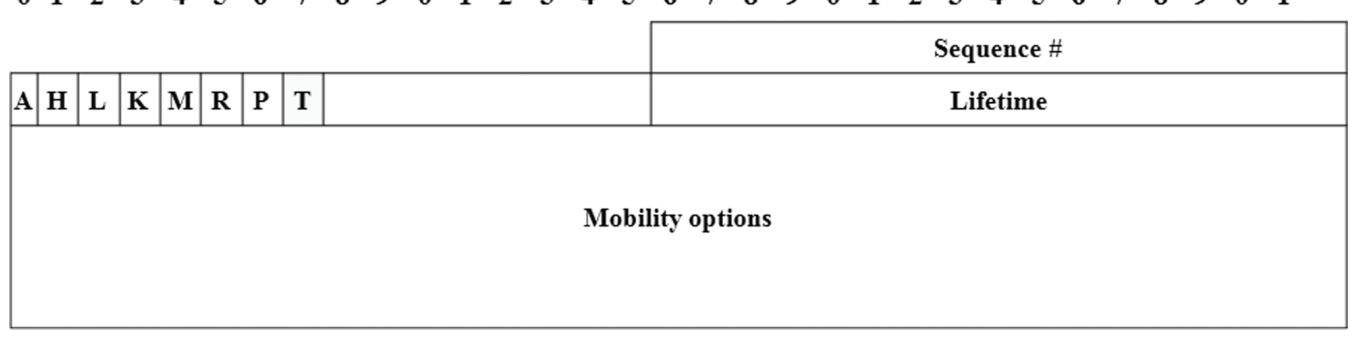

Fig. 5. PBU message expansion for PMIPv6.
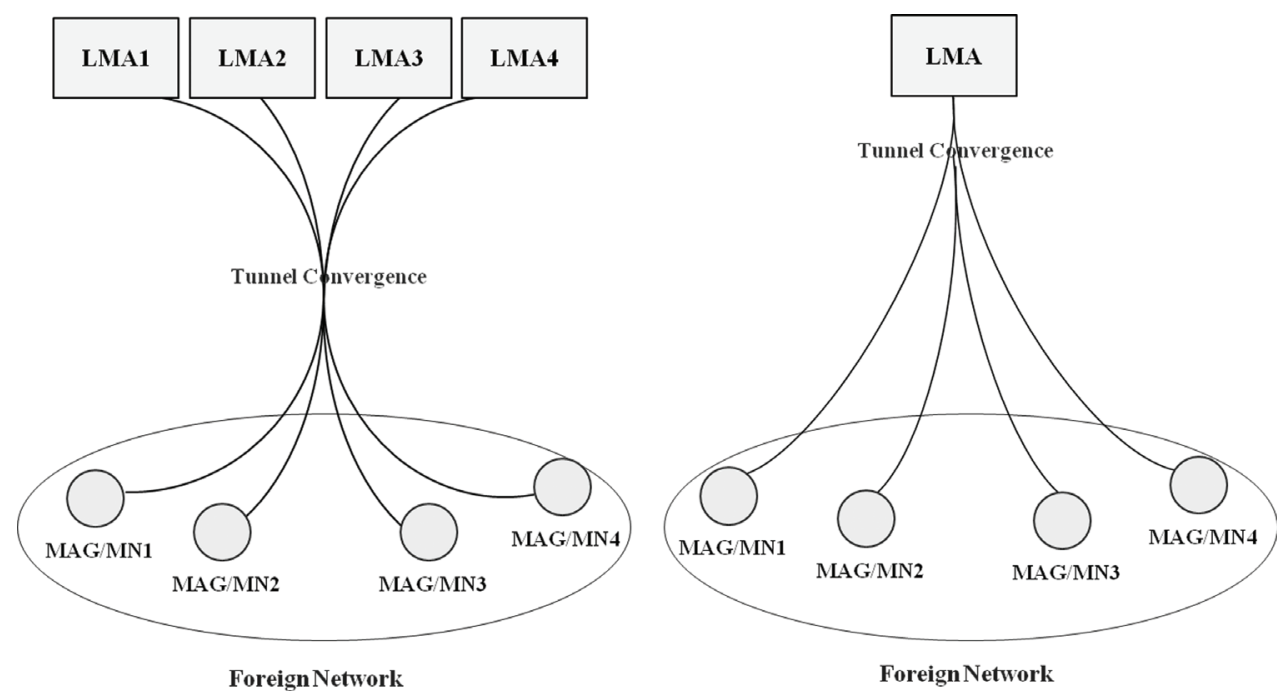

Fig. 6. Tunnel convergence.

router, to send out MLD, via the 'stream-interface' bi-directional tunnel. Note that LFH concerns 'Binding Cash Lifetime' in maintenance of the multicast state of MN. MN, upon mobility, is to send out MLD completion messages via the tunnel, while LMA and MN, during the course of handover, are not to trade any messages, apart from PMIPv6 signaling. Note that MN's mobility does not affect the multicast state of packets.

Figure 4 shows streamlined MLD proxying, demanding preparation and maintenance of the three different information scopes; MAG/MN, home address and COA of MAG/MN, and the current AR address and MAG/MN binding lifetime/Group membership list of MAG/MN, such as multicast address, mode, scope, etc./Forward list listing forwarding interfaces of every multicast group. Figure 5 below shows message expansion of the Proxy Binding Update (PBU) of the proxy mobile IPv6 protocol message. PBU is to be sent out from MAG to LMA, by adding a flag (T) for the expanded mobility option. Refer to the following Chapter 3.3 for the role of T Flag, improving tunnel convergence.

As MAG is to administer the multicast group membership of the concerned MN, MN upon mobility is to be subject to the reorganization of multicast tree or tunneling update. Note that the LFH method modifies the MLD host function by means of address reference (such as Trigger) for continual multicast. MAG works as MLD to receive MLD Reports out of the concerned MN, to hand over to LMA. 


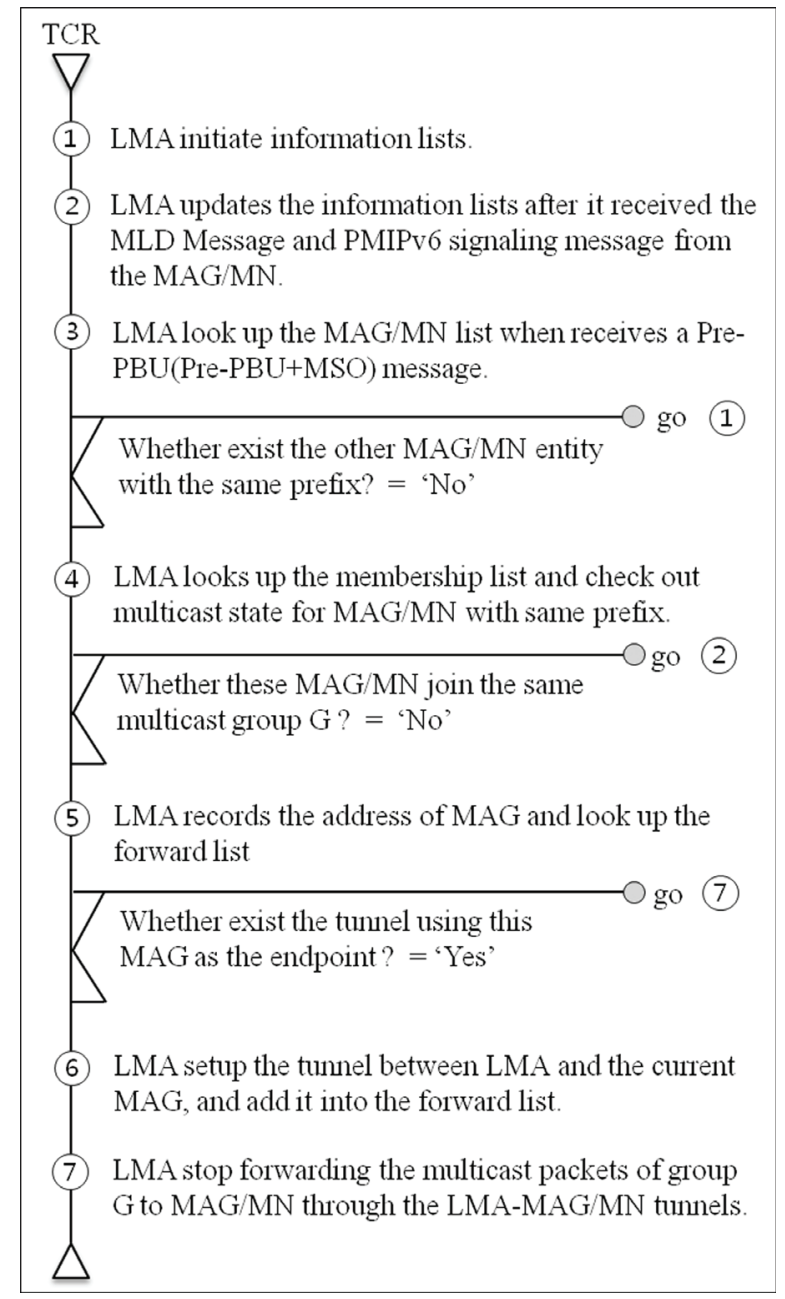

Fig. 7. below details a TCR algorithm.

\subsection{TCR (tunnel combination and reconstruction) algorithm}

Multicast via bi-directional tunneling is to be done by multiple unicasts, thereby set to multicast identical packets to multiple tunnels, by the amount equal to the concerned MNs. Figure 6 shows the two different tunnel convergences: MAG/MN out of the linkage of the identical multicast groups through which LMA packets travel/Multiple MAG/MNs out of the same multicast group upon access to the external network (such as LMA). A Designated Multicast Service Provider (DMSP) [3] is to be incorporated for Mobile Multicast Protocol, to resolve tunnel convergence and designate a single LMA in conversion of the algorithm from the left to the right one of the figure.

The TCR algorithm is commenced by LMA, listing information, receiving MLD and PMIPv6 signaled out of MAG/MN, and updates the information listed. LMA, upon reception of Pre-PBU (Pre-PBU + MSO), refers to MAG/MN, checks for the state multicast for the prefix MAG/MN, and, in any cases MAG being End Point, adds tunnel and forward list between the concerned LMA and the current MAG. LMA thereby halts forwarding of Group G packets via MAG/MN tunnel. 


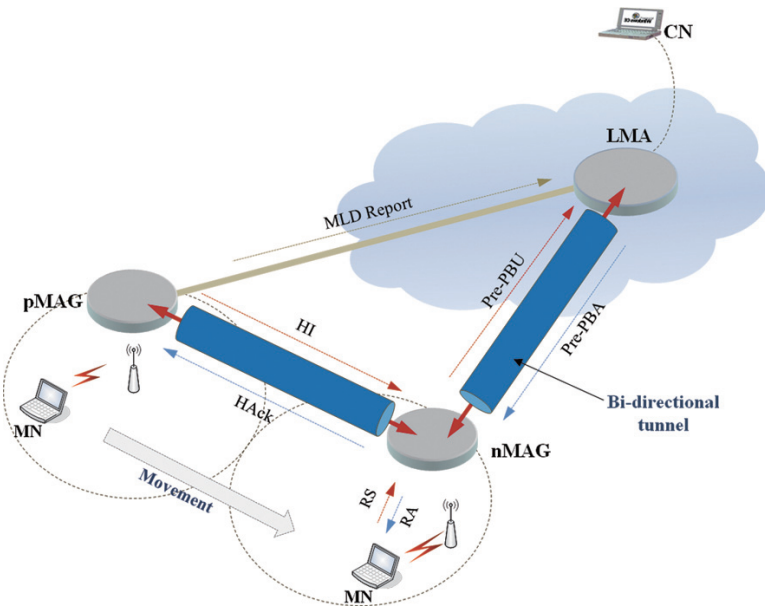

(a)

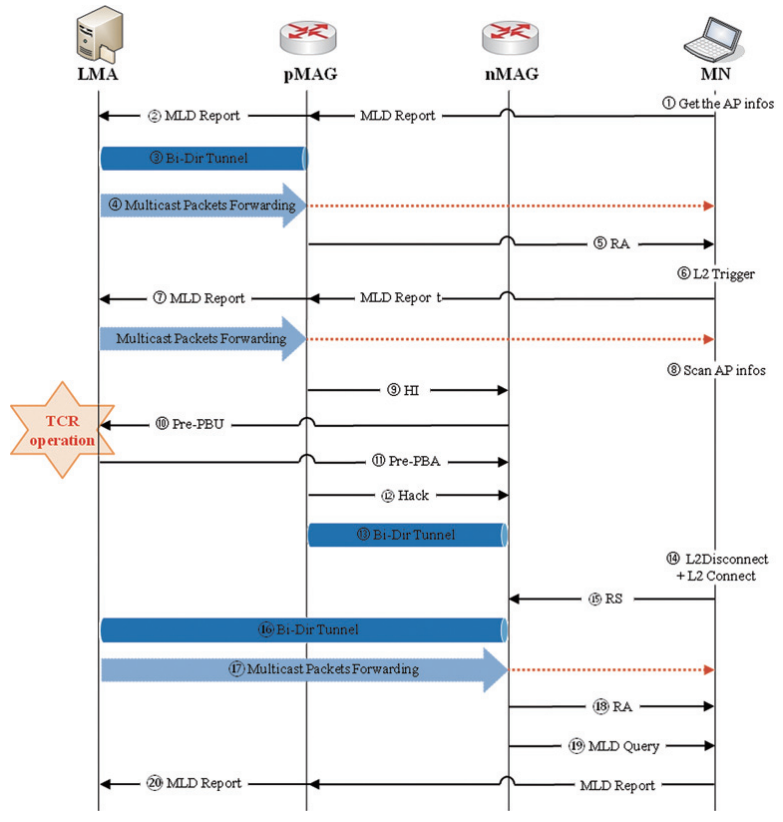

(b)

Fig. 8. (a) Intra-domain LFH sequence for LMA, (b) Intra-domain flow.

\subsection{Operational sequence}

Multicast in PMIPv6 network is to be done by MAG and LMA handling of the MN grouping. MAG and LMA hand over the multicast data towards MN, being entitled to services. Figure 8(a) shows how LFH works, the intra-domain of LMA, and multicast handover. Almost identical, this handover incorporates Pre-PBU (Previously-PBU) and Pre-PBA (Previously-PBA) to be traded between LMA and nMAG for Proxy Binding to be done earlier. The pMAG, upon recognition of MN's handover, sends out HI to nMAG for nMAG and LMA to be able to execute the Proxy Binding not later than when nMAG sends out Hack, which significantly reduces multicast loss.

Figure 8(b) below shows the Intra-domain Flow of LFH for LMA. The multicast is to be commenced upon reception of AP (Available Access Point) information (1) and MLD send-out (2). Upon initial access of MN to PMIPv6 network, a bi-directional tunnel between pMAG and LMA is to be set up for the binding of MN (3), thereby being entitled to multicast service (4). The mobility of MN is to be tracked MAG, sending out Router Advertisement (RA) (5). Upon mobility of MN from pMAG to nMAG, pMAG becomes able to detect MN access (6), with use of L2 Trigger, while the pMAG sends out MLD Report to LMA (7). pMAG is to search APs in the vicinity (8) and send out HI, comprising but not limited to MNID, LMAA, Multicast LMA, and Multicast state (9), while nMAG queries Pre-PBU to LMA for binding process (10. LMA, upon reception of Pre-PBU, is to run over MAG/MN list, as part of the operational procedure of TCR Algorithm LMA is to hand over Pre-PBA (11), while nMAG sends out Hack to pMAG for acceptance (or rejection) (12. Upon reception of Hack, the pMAG is to set up a bi-directional tunnel between pMAG and nMAG (13) through which packets travel. Note that any packets addressed to MN are to be by way of pMAG, nMAG, and LMA. MN, upon detection of L2 access (or disconnection) (14), is to send out Router Solicitation (RS) to nMAG as MN gets into the PMIPv6 domain (15). Note that Source Address field of RS contains the home address of MN or, if unavailable as MN accesses for the 


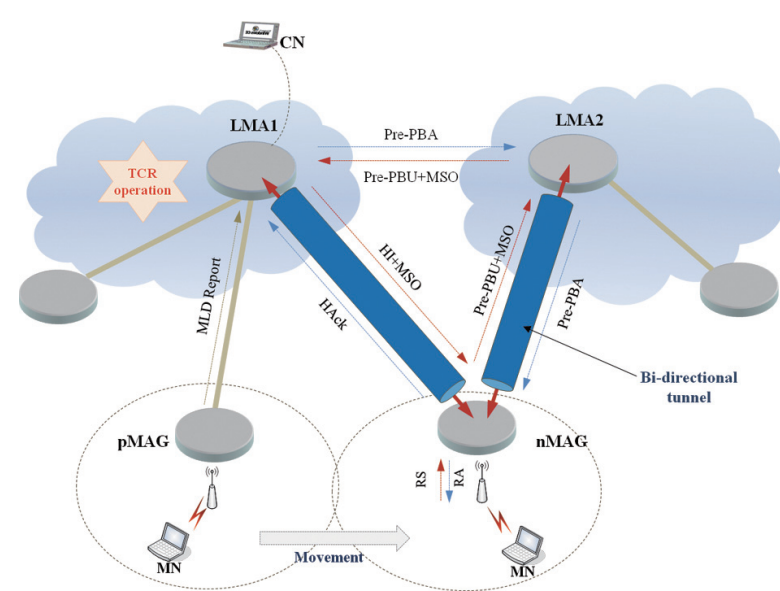

(a)

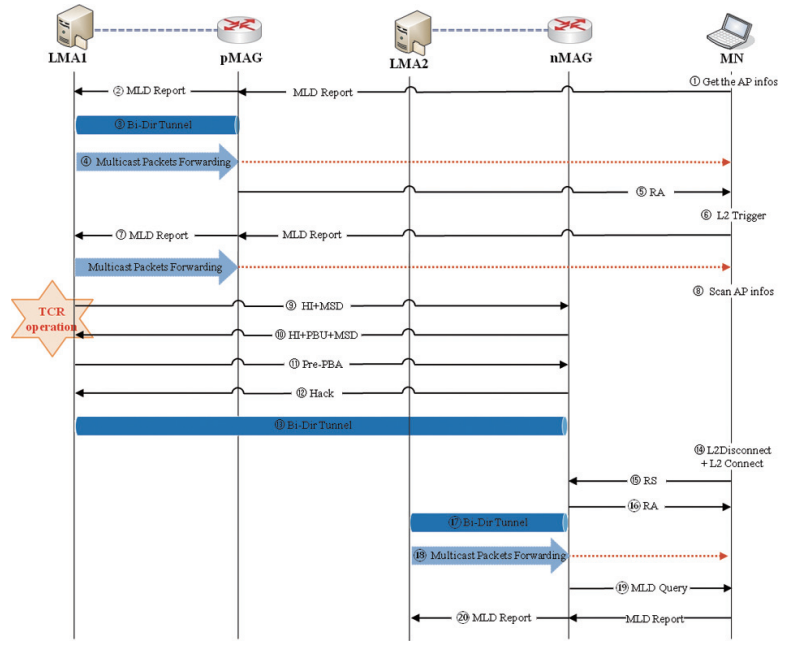

(b)

Fig. 9. (a) Inter-domain LFH sequence for LMA, (b) Inter-domain flow.

first time, the unspecific address. Upon reception of RS by nMAG, Proxy Binding is to be performed via the bi-directional tunnel between LMA and nMAG (16), thereby multicasting to MN upon reception of packets (17). nMAG, for the continued track-down of MN mobility is to send out Router Advertisement (RA) (18), nMAG is to query MLD to MN to confirm the multicast request, upon completion of location registration (18). MN, initially accessing PMIPv6, is to send out MLD Report to the concerned MAG for group membership (20), thereby being entitled to the multicast services.

Note that PMIPv6 is only for intra-domain mobility as designed for the local mobility protocol. Figure 9(a) below shows inter-domain mobility by LMA-MAG tunneling multicast handover. Inter-domain multicast handover is to be done the same as PMIPv6. Almost identical to that of the intra-domain, the inter-domain handover, prior to nMAG sending out Hack to LMA 1, incorporates LMA1 and nMAG trading Pre-PBU (Previously-PBU), Pre-PBA (Previously-PBA), and MSOs for Proxy Binding to be done earlier:

Figure 9(b) shows the Inter-domain Flow of LFH for LMA. The multicast is to be commenced upon recognition of $\mathrm{MN}$ and obtainment of information, all done by L2 Trigger. Upon transferal of L2 Trigger to pMAG, MN is to commence handover from pMAG to nMAG (6), as well as send out MLD Report, as done for Intra-domain. (7). pMAG is to search APs in the vicinity (8) and send out HI, comprising but not limited to MN-ID, HNP of MN, address of LMA of MN, Multicast Support Option (MSO) to nMAG (9), while nMAG queries Pre-PBU + MSO to LMA2 for multicast membership information of $\mathrm{MN}$, containing MSO as well (10). LMA2 upon reception of the information is to authenticate and arrange connection of domain(s) handing over Pre-PMA to pMAG (12. pMAG, upon reception of Hack is to set up a bi-directional tunnel between pMAG and nMAG (13) while sending out multicast, together with L2 Connection Notification, to the concerned (14). MN is to send out Router Solicitation (RS), containing the home address of MN. The nMAG, for the continued track-down of MN mobility is to send out Router Advertisement (RA) (16). Note that Proxy Binding has previously done (17), thereby allows LMA2 to multicast to MN upon the RS and RA done (18). Upon completion of location registration, nMAG is to query MLD to MN to confirm the multicast request (19). MN, initially accessing PMIPv6, is to send out MLD Report to the concerned MAG for group membership, thereby being entitled to the multicast services (20). 
Table 1

Parameters for network evaluation

\begin{tabular}{clc}
\hline Parameter & Description & Value \\
\hline$N$ & Number of MN in group & $1-20$ \\
$M$ & Number of multicast group & $1-20$ \\
$r$ & Ratio of multicast source & $64-512$ \\
$P$ & Number of source in group & 1 \\
$L s$ & Size of multicast packet & 1378 \\
$n$ & Number of available AP & 7 \\
\hline
\end{tabular}

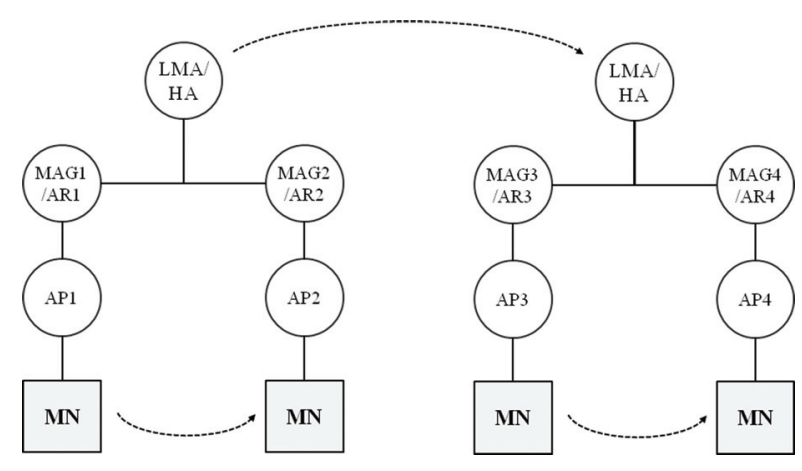

Fig. 10. Network model.

\section{Performance analysis}

The performance is evaluated using mathematical modeling of the delay time and cost analysis for the multicast handover process. Figure 10 depicts the reference network model. The number of hope is two times, which are between LMA/HA and MAG1/AR1 and between LMA/HA and MAG2/AR2, while the number of hope between MN and AP and between AP and MAG/AR is one, respectively. The communication path between LMA/HA and LMA/HA, between LMA/HA and MAG/AR is cable, while the one between MAG/AR and MN is wireless.

A derived equation of the communication delay model can be used for calculating the delay $D_{X-Y}[30]$.

$$
\begin{aligned}
& T_{W-R T}(h, k)=3.63 k+3.21(h-1) \\
& T_{W L-R T}(h, k)=17.1 k
\end{aligned}
$$

$K$ is the length of KB unit packet, $H$ is the number of hope, and $T_{W-R T}$ and $T_{W L-R T}$ is the roundtrip time of the cable link and wireless link, respectively. The result of the multicast broken time is used for estimating the protocol cost. Table 1 summarizes the parameters for network evaluation.

\subsection{Multicast delay time}

The multicast delay time means the time that MN cannot receive the multicast packet during handover. It consists of handover delay of the L2 and IP layer and the process of the scan $\left(t_{\text {scanning }}\right)$, authentication, and connection $\left(t_{A A}\right)$. Delay time consists of transfer searching time $\left(t_{M D}\right)$, address setting time $\left(t_{D A D}\right)$, location update time to HA and $\mathrm{CN}$. The transfer search time depends on the transmit interval of the RA non storage address organization, and permits 30-70 m RA interval. Address organization time takes $1000 \mathrm{~ms}$ according to the neighbor discovery protocol, and depends upon the DAD (duplicate address detection) process. The location update time of $\mathrm{MN}$ and $\mathrm{HA}$ is indicated in $R T T(M N, H A)$, and depends on roundtrip time. Figure 11 shows the multicast delay time of BT, RS, MFH, LMM and LFH methods.

In the case of the BT method, MN does not rejoin the multicast group, and the greater part of the multicast delay time is the handover delay time of L2 and IP. Therefore, the scanning time $\left(t_{\text {scanning }}\right)$, the authentication and connect time $\left(t_{A A}\right)$, transfer search time $\left(t_{M D}\right)$, address setting time $\left(t_{D A D}\right)$, and location update time $R T T(M N, H A)$ () are included. Thus, the multicast delay time of BT is given by the following equation.

$$
t_{B T}=t_{\text {sanning }}+t_{A A}+t_{M D}+t_{D A D}+R T T(M N, H A)
$$


I. Im and J. Jeong / Cost-effective and fast handoff scheme in proxy mobile IPv6 networks with multicasting support 297

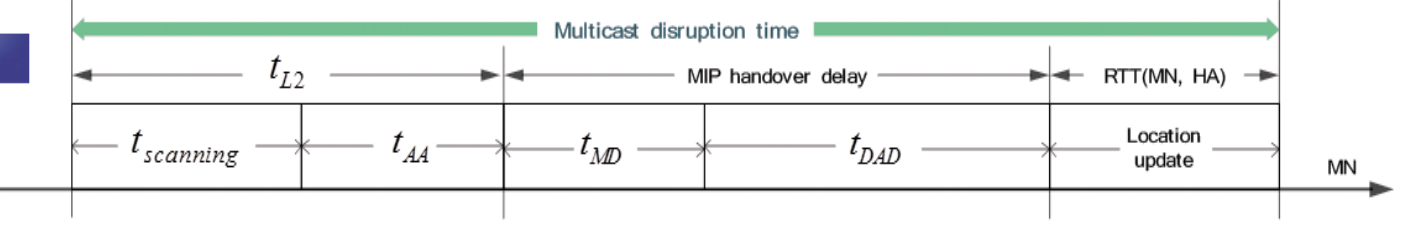

RS

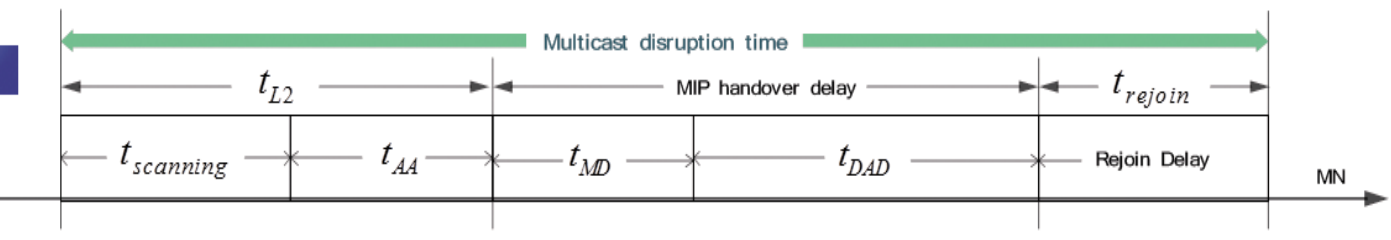

MFH

Multicast disruption time

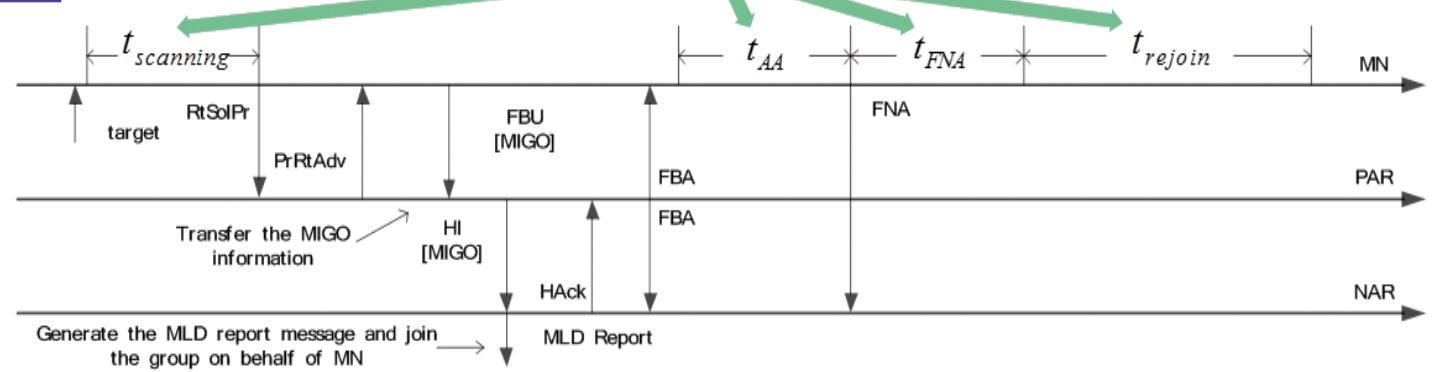

LMM

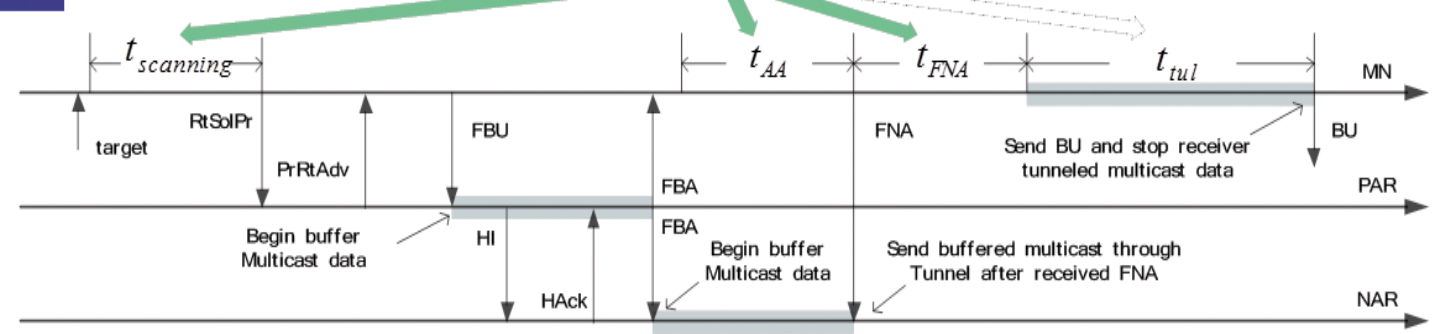

LFH

Multicast disruption time

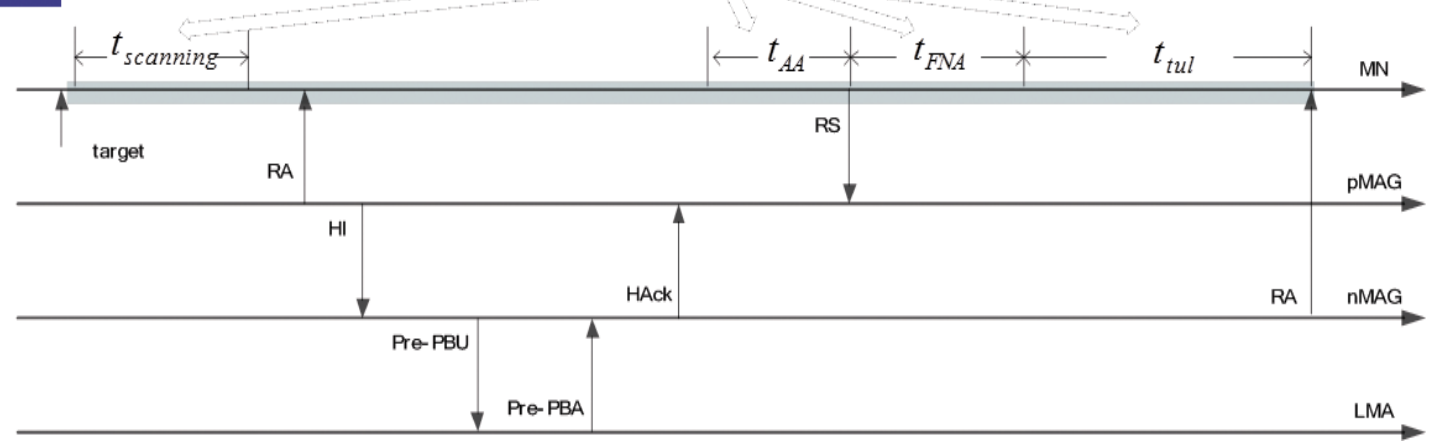

Fig. 11. Multicast delay time of the BT, RS, MFH, LMM and LFH method. 
Table 2

Size of signaling message

\begin{tabular}{llc}
\hline Message & Usage & Size \\
\hline RS & ICMPv6 & $16 / 8$ \\
RA & ICMPv6 & 64 \\
NA & ICMPv6 & $32 / 24$ \\
FNA & ICMPv6 & 24 \\
BU & MIPv6 & 56 \\
BA & MIPv6 & 40 \\
RtSolPr & FMIPv6 & $24+16 \mathrm{n}$ \\
PrRtAdv & FMIPv6 & 104 \\
HI/HI + MSO & FMIPv6/PMIPv6 & $72 / 80$ \\
Hack & FMIPv6/PMIPv6 & 32 \\
FBU & FMIPv6 & 72 \\
FBack & FMIPv6 & $32 / 12$ \\
PBU/Pre-PBU/Pre-PBU + MSO & PMIPv6 & $56 / 40 / 48$ \\
PBA/Pre-PBA & PMIPv6 & 4028 \\
MLD report & MLD & $16+16 \mathrm{~m}$ \\
\hline
\end{tabular}

In the case of the RS method, the subscriber rejoins the multicast group during reentrance delay time $\left(t_{\text {rejoin }}\right)$ after $\mathrm{MN}$. Therefore, the scanning time $\left(t_{\text {scanning }}\right)$, the authentication and connect time $\left(t_{A A}\right)$, transfer search time $\left(t_{M D}\right)$, and address setting time $\left(t_{D A D}\right)$ contain the reentrance delay time $\left(t_{\text {rejoin }}\right)$. Thus, the multicast delay time of RS method is given by the following equation:

$$
t_{R S}=t_{\text {sanning }}+t_{A A}+t_{M D}+t_{D A D}+t_{\text {rejoin }}
$$

This depends on the MLD query delay interval, and can exceed $60 \mathrm{~s}$, in the case that the interval of query is $125 \mathrm{~s}$ [28]. MN moves to a new subnet in the case of sending an unneeded MLD Report message, and depends on the delay of the return multicast tree and the message delivery delay among multicast group components.

In the case of the MFH method, multicast packets are transmitted in the current AR, based on the RS method. MN sets the multicast state using a FBU and HI message in advance, for reducing reentrance delay time, when it moves from PAR to NAR. Therefore, the multicast delay time includes the scanning time $\left(t_{\text {scanning }}\right)$, the authentication and connect time $\left(t_{A A}\right)$, the IP reconnect time $\left(t_{F N A}\right)$, and the reentrance delay time $\left(t_{\text {rejoin }}\right)$, as the following equation:

$$
t_{M F H}=t_{\text {sanning }}+t_{A A}+t_{F N A}+t_{\text {rejoin }}
$$

From then, NAR sends the MLD Report message in advance in order to reduce the reentrance delay time. Therefore, the multicast delay time of MFH method is less than RS. The multicast delay time includes the scanning time $\left(t_{\text {scanning }}\right)$ before IP reconnect time $\left(t_{F N A}\right)$, and authenticate and connect time $\left(t_{A A}\right)$, as the following equation:

$$
t_{L M M}=t_{\text {sanning }}+t_{A A}+t_{F N A}
$$

The suggested LFH method does not include the scanning time and the authentication and connect process. In addition, it can get the multicast packet without delay during handover, because it executes PBU process in advance, as in the following equation:

$$
t_{L F H}=0
$$

Therefore, we can estimate the multicast delay time in the same conditions using mathematical analysis, 
and the RS method takes a long time, while the suggested LFH method takes a short time, in accord with the multicast delay time, as follows:

$$
t_{L F H}<t_{L M M}<t_{B T}<t_{M F H}<t_{R S}
$$

\subsection{Cost analysis}

The protocol cost consists of the signaling cost (CS) and packet delivery cost (CD). This means the sum of the signaling message and packet delivery cost in handover of delivery cost. Here, we estimate the cost of protocol, using the hope based delay analysis method [29]. Suppose that the multicast source ratio is $\lambda_{s}$ (packet/s), the multicast packet size, transmission rate of RA, tunnel transmission time, and delivery delay between $X$ and $Y$ is $L_{s}$ (byte), $\lambda_{r}$ (packet/s), $t_{t n l}(\mathrm{~S})$, and $D_{X-Y}(\mathrm{~S})$, respectively. Table 2 shows the size of the signaling message. In the table, $n$ is the number of scanned AP by MN, while $m$ is the number of the MN joined multicast group.

\subsubsection{Signaling cost}

The signaling cost is defined as the necessary cost during the multicast procedure when MN handover occurs. Signaling cost estimates using the size of signaling messages. The signaling cost of the intradomain LMM method can be calculated using the multicast delay time of the LMM method, and the multicast source ratio between MN and AR. The signaling process between MN and PAR is that MN send RtSolPr and FBU message to PAR, and vice versa, PrRtAdv and FBack message. In the case of between PAR and NAR, HI, Hack, and FBack signaling messages are exchanged, while MN only sends an FNA message to NAR between MN and NAR. Also, BU, BA, and MLD Report signaling messages are exchanged between MN and HA. Therefore, the signaling cost of the intra-domain LMM method is as follows.

$$
\begin{aligned}
C S_{L M M}^{I N T R A}= & 64 \times\left(\left\lfloor\frac{t_{L M M}}{\lambda_{S}}\right\rfloor+1\right) \times D_{M N-A R}+(212+16 n \pm 20) \times D_{M N-P A R}+(116 \pm 20) \\
& \times D_{P A R-N A R}+24 \times D_{M N-N A R}+(112+16 m) \times D_{M N-H A}
\end{aligned}
$$

The signaling cost of the intra-domain LFH method can be calculated using the multicast delay time of LFH between MN and MAG, and the rest are the same as the LMM method. The MLD Report message is exchanged between MN and pMAG, and between pMAG and LMA, while the HI and HAck are exchanged between pMAG and nMAG. On the other hand, MN sends an RS message to nMAG between MN and nMAG, while Pre-PBU and Pre-PBA signaling messages are exchanged between nMAG and LMA. Therefore, the signaling cost of the intra-domain LFH method is as follows:

$$
\begin{aligned}
C S_{L F H}^{I N T R A}= & 64 \times\left(\left\lfloor\frac{t_{L F H}}{\lambda_{S}}\right\rfloor+1\right) \times D_{M N-M A G}+(16+16 m) \times D_{M N-p M A G}+104 \\
& \times D_{p M A G-n M A G}+8 \times D_{M N-n M A G}+68 \times D_{n M A G-L M A}+(16+16 m) \times D_{p M A G-L M A}
\end{aligned}
$$

The signaling cost of the intra-domain LMM method is the same as the signaling cost of the interdomain LMM method:

$$
C S_{L M M}^{I N T E R}=C S_{L M M}^{I N T R A}
$$

The signaling cost of the intra-domain LFH method is equal to the case of the inter-domain between MN and MAG, and a MLD Report message is exchanged between MN and pMAG and between nMAG and LMA2. LMA1 exchanges an HI + MSO message and HAck message with nMAG. Also, an RS 
message is exchanged between MN and nMAG, while Pre-PBU + MSO and Pre-PBA messages are exchanged between nMAG and LMA2, and between LMA1 and LMA2.

$$
\begin{aligned}
C S_{L F H}^{I N T E R}= & 64 \times\left(\left[\frac{t_{L F H}}{\lambda_{S}}\right]+1\right) \times D_{M N-M A G}+(16+16 m) \times D_{M N-p M A G}+112 \times D_{L M A 1-n M A G} \\
& +8 \times D_{M N-n M A G}+76 \times D_{n M A G-L M A 2}+(16+16 m) \times D_{p M A G-L M A 1}+76 \times D_{\text {LMA1-LMA } 2}
\end{aligned}
$$

\subsubsection{Packet delivery cost}

Packet delivery cost means the necessary cost when MN sends all packets. This is calculated using the number of the MN joined multicast group, the ratio of the multicast source, the size of the multicast packet, the multicast delay time, the number of each hope, and the value of the tunnel transmission time.

The delivery cost of the inter-domain LMM method can be calculated by the packet delivery cost of HA-PAR, PAR-NAR, and NAR-MN, as the following equation:

$$
C D_{L M M}^{I N T R A}=m \lambda_{S} L_{S} t_{L M M} D_{H A-P A R}+\left(1+\frac{40}{L_{S}}\right) m \lambda_{S} L_{S} t_{t n l}\left(D_{P A R-N A R}+D_{N A R-M N}\right)
$$

In the case of the delivery cost of the inter-domain LFH method, the cost of LMA-pMAG is not included, because the multicast delay time is 0 . The following equation depicts the packet delivery cost of pMAG-nMAG and nMAG-MN:

$$
C D_{L F H}^{I N T R A}=\left(1+\frac{40}{L_{S}}\right) m \times \lambda_{S} L_{S} t_{t n l}\left(D_{p M A G-n M A G}+D_{n M A G-M N}\right)
$$

The delivery cost of the intra-domain LMM method is the same as the one of the inter-domain LMM method:

$$
C D_{L M M}^{I N T E R}=C D_{L M M}^{I N T R A}
$$

The delivery cost of the intra-domain LFH method is the same as the one of the inter-domain LFH method, and uses the packet delivery cost of the LMA1-nMAG and nMAG-MN for calculation:

$$
C D_{L F H}^{I N T E R}=\left(1+\frac{40}{L_{S}}\right) m \times \lambda_{S} L_{S} t_{t n l}\left(D_{L M A I-n M A G}+D_{n M A G-M N}\right)
$$

\subsubsection{Total cost}

The cost of the BT method is less than the RS and MFH methods, because it is simple and need not explain the size of the signal message. The total cost of the inter-domain BT method is given by the following equation:

$$
\begin{aligned}
C_{B T}^{I N T R A}= & C S_{B T}^{I N T R A}+C D_{B T}^{I N T R A}=64 \times\left(\frac{t_{B T}}{\lambda_{r}}+1\right) \times D_{M N-A R}+(112+16 m) \times D_{M N-H A} \\
& +m \lambda_{r} t_{B T} L_{S} D_{H A-A R}
\end{aligned}
$$

The delivery cost of the RS method is increasing, due to the long join delay time. The total cost of the inter-domain RS method is given by the following equation:

$$
\begin{aligned}
C_{R S}^{I N T R A}= & C S_{R S}^{I N T R A}+C S_{R S}^{I N T R A}=64 \times\left(\frac{t_{R S}}{\lambda_{r}}+1\right) \times D_{M N-A R}+96 \times D_{M N-H A}+(16+16 m) \\
& \times D_{M N-A R}+m \lambda_{S} t_{R S} L_{S} D_{A R-M N}
\end{aligned}
$$

The multicast delay time of the MFH method is long, and the protocol cost is also high, due to the size of the signaling message. The total cost of the inter-domain MFH method is given by the following equation: 


$$
\begin{aligned}
C_{M F H}^{I N T R A}= & C S_{M F H}^{I N T R A}+C D_{M F H}^{I N T R A}=(232 \pm 20+16 n) \times D_{M N-P A R}+(136 \pm 20) \\
& \times D_{P A R-N A R}+24 \times D_{M N-N A R}+m \lambda_{S} L_{S} t_{M F H} D_{P A R-M N}
\end{aligned}
$$

The multicast delay time of the LMM method can be reduced, because the HA sends a multicast packet based on the BT method. Therefore, the cost of the LMM method is less than the BT one. The total cost of the inter-domain LMM method is the sum of the signaling cost calculated above, and the packet delivery cost:

$$
C_{L M M}^{I N T R A}=C S_{L M M}^{I N T R A}+C D_{L M M}^{I N T R A}
$$

The signaling cost and packet delivery cost for the LFH method is less than other methods, because there is no multicast delay time, as follows:

$$
C_{L F H}^{I N T R A}=C S_{L F H}^{I N T R A}+C D_{L F H}^{I N T R A}
$$

The total cost of the intra-domain BT method is the same as the inter-domain one, as follows:

$$
C_{B T}^{I N T E R}=C_{B T}^{I N T R A}
$$

The total cost of the intra-domain RS method is the same as the inter-domain one, as follows:

$$
C_{R S}^{I N T E R}=C_{R S}^{I N T R A}
$$

The total cost of the intra-domain MFH method is the same as the inter-domain one, as follows:

$$
C_{M F H}^{I N T E R}=C_{M F H}^{I N T R A}
$$

The total cost of the intra-domain LMM method is the sum of the signaling cost calculated above, and the packet delivery cost:

$$
C_{L M M}^{I N T E R}=C S_{L M M}^{I N T E R}+C D_{L M M}^{I N T E R}
$$

The total cost of the intra-domain LFH method is the sum of the signaling cost, and the packet delivery cost is the same as the LMM method:

$$
C_{L F H}^{I N T E R}=C S_{L F H}^{I N T E R}+C D_{L F H}^{I N T E R}
$$

\subsection{The result of numerical analysis}

Comparing the protocol cost of the existing method with that of the LFH method, using the multicast source ratio $256 \mathrm{~kb} / \mathrm{s}$, Fig. 12 depicts the protocol cost according to the number of MN in the interdomain multicast group, while Fig. 13 shows the protocol cost of the intra-domain. The RS method incurred the delivery cost, due to the long join delay time. The MFH method also has high cost, because of the long multicast delay time, and the extension of the signaling message. In contrast, the BT method has less cost than the MFH and RS methods, because it is simple, and has no extension of signaling. The cost of the LMM method is less than the BT method, because HA sends the multicast packet based on the BT method. Finally, we can verify that the suggested LFH method is definitely less than the other methods, because there is no multicast delay time. 


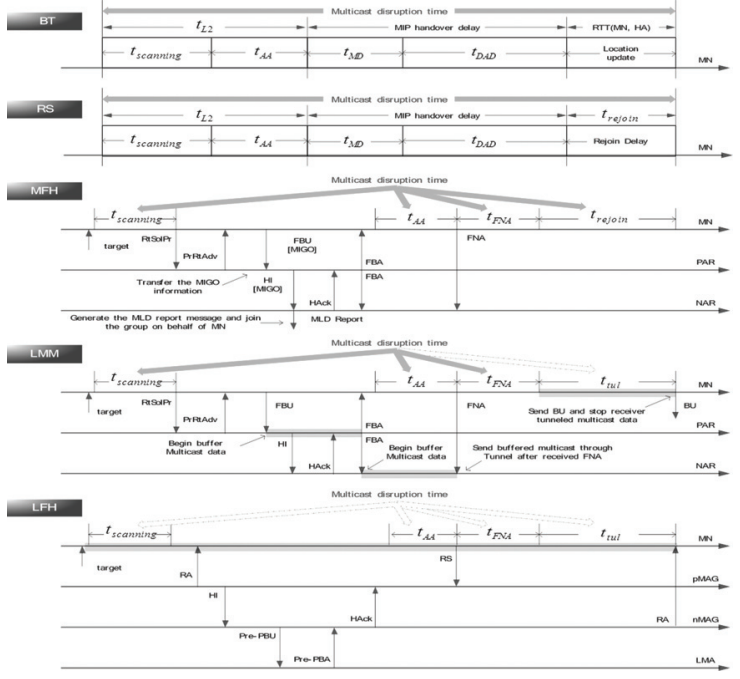

Fig. 12. The protocol cost, according to the number of MN in the inter-domain multicast group.

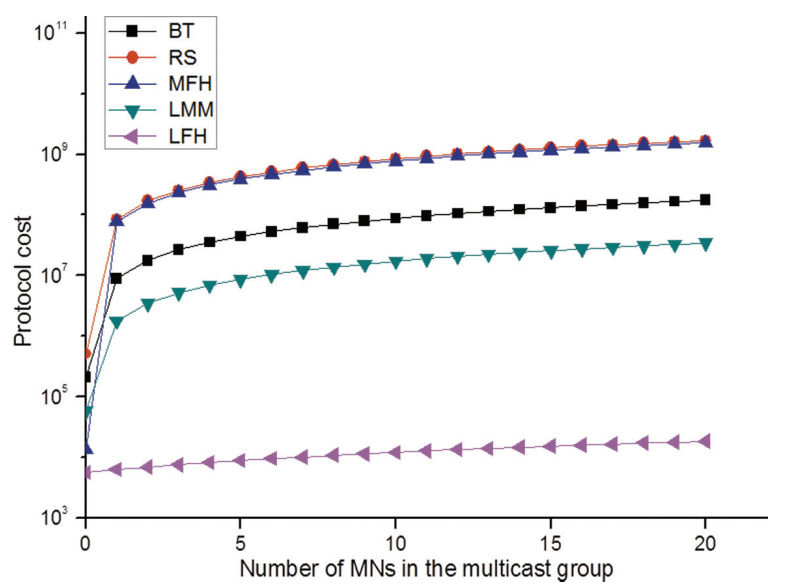

Fig. 14. The ratio of the inter-domain multicast source $(\mathrm{kb} / \mathrm{s})$.

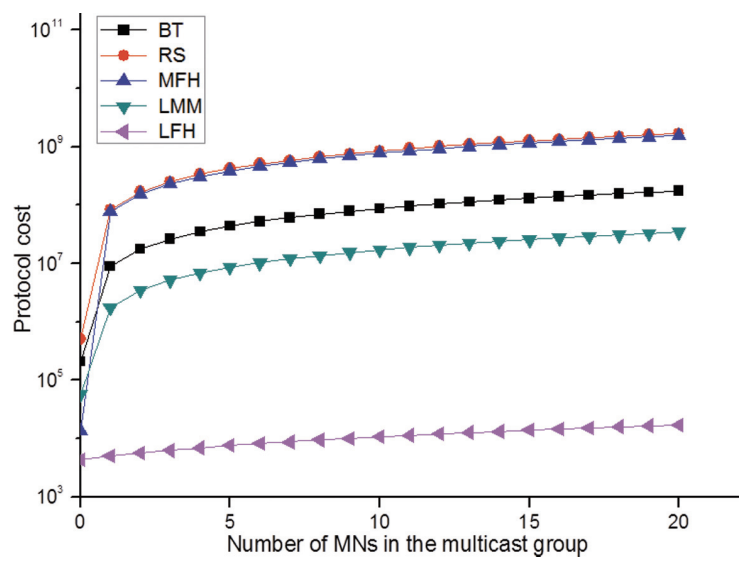

Fig. 13. The protocol cost, according to the number of $\mathrm{MN}$ in the intra-domain multicast group.

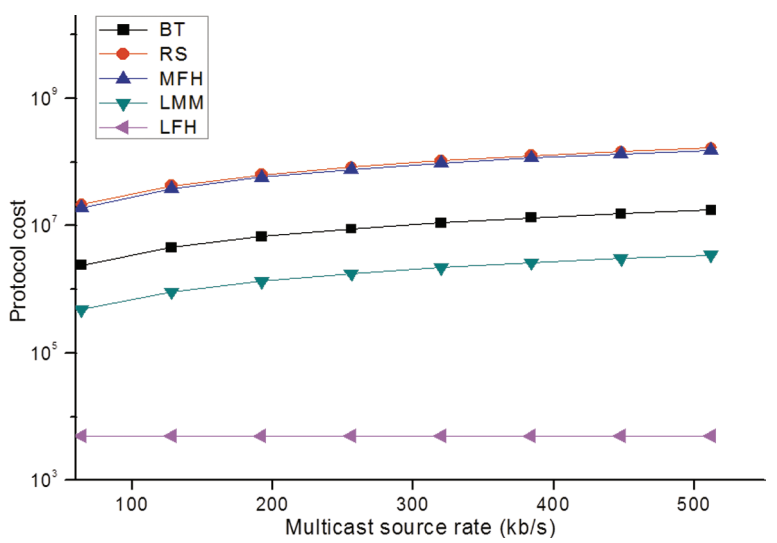

Fig. 15. The ratio of the intra-domain multicast source $(\mathrm{kb} / \mathrm{s})$.

Figure 14 depicts the protocol cost according to the inter-domain multicast source rate, while Fig. 15 shows the intra-domain protocol cost. In the case of the existing method, increased protocol cost will lead to an increasing multicast source rate. However, the suggested LFH method has a constant protocol cost in all multicast source rates, and also, low cost.

Figure 16 depicts the protocol cost according to the number of tunnels, if the tunnels are inter-domain converged, while Fig. 17 shows the intra-domain protocol cost. If the suggested TCR algorithm is used, the protocol cost can be compared, according to the use of the TCR algorithm. In the case of the existing method, the high cost occurs as the number of converged tunnels increases. The LFH method, which does not use the TCR algorithm, and the LMM method are based on a unicast tunnel, which transmits multicast packets, and constructs many unicast tunnels for sending many copies of multicast data, when MNs of identical multicast group connect with AR. As a result, the protocol cost is increased. In the case of BT, RS, and MFH methods, there is no problem of tunnel convergence when the current AR sends the 

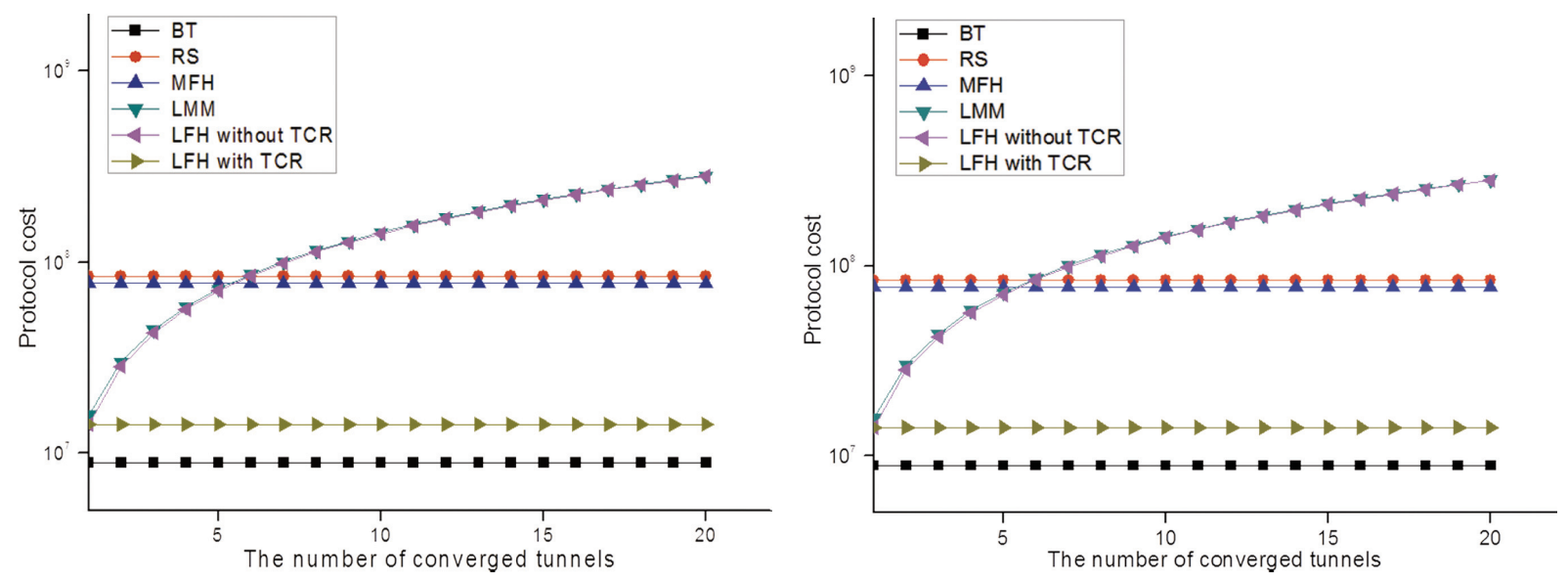

Fig. 16. The number of converged tunnels of the intra-domain. Fig. 17. The number of converged tunnels of the intra-domain.

multicast packet. The LFH method also has a problem with tunnel convergence, because it is based on a unicast tunnel, which transmits multicast packets. However, if the TCR algorithm is applied to the LFH method, the protocol cost will be decreased. Therefore, the tunnel convergence problem can be solved, using the TCR algorithm.

\section{Conclusion}

PMIPv6 is a protocol based on unicast transmission, which is the most basic communication method. In the existing PMIPv6, packet loss is incurred by handover delay, because the packet is sent by creating a tunnel between LMA and MAG, after the end of handover. In addition, there is a tunnel convergence problem, due to inefficient usage of network resources between LMA and MN. To support efficient multicast service for mobile devices, a PMIPv6 network based LFH method is suggested in this paper. The LFH method develops an MLD proxy function simplified LMA for decreasing the operational overhead of LMA, and a modified MLD host function for reducing delay time. Therefore, the operational overhead is decreased, and can maintain the multicast state. The suggested method eliminates service cessation, and prevents packet loss, by accomplishing a proxy binding procedure in advance. In addition, it solves the tunnel convergence problem, using the TCR algorithm. Through analyzing the multicast delay time, the LFH method is less than the other methods, in accordance to the multicast delay time. In the case of analyzing the calculating cost, the LFH method also show excellent performance.

With the lower cost, a faster mobility management scheme is needed, because it carries out the vertical handoff of various heterogeneous networks efficiently in a future mobile environment, which includes a huge multimedia services need. Therefore, based on this result, the study scope will be expanded for supporting seamless handover and fast mobility.

\section{Acknowledgments}

This research was supported by Basic Science Research Program through the National Research Foundation of Korea(NRF) funded by the Ministry of Education, Science and Technology(2011-0027030). Corresponding author: Jongpil Jeong. 


\section{References}

[1] S. Deering, W. Fenner and B. Haberman, Multicast Listener Discovery (MLD) for IPv6, RFC 2710, October 1999.

[2] D. Johnson, C. Perkins and J. Arkko, Mobility Support in IPv6, RFC 3775, June 2004.

[3] T. Harrison, C. Williamson, W. Mackrell and R. Bunt, Mobile multicast (MoM) protocol: Multicast support for mobile hosts, in: Proceedings of ACM/IEEE MOBIGCOM'97 (1997), 151-160.

[4] R. Lin and K. Wang, Mobile multicast support in IP networks, INFOCOM2000, (2000), 1664-1672.

[5] Y.J. Suh, H.S. Shin and D.H. Kwon, An efficient multicast routing protocol in wireless mobile networks, Kluwer Wireless Networks 7(5) (2001), 443-453.

[6] R. Koodli et al., Fast handover for mobile IPv6, RFC 4068, July 2005.

[7] H. Soliman, C. Castelluccia, K. El Malki and L. Bellier, Hierarchical mobile IPv6 mobility management (HMIPv6), RFC 4140, August 2005.

[8] S. Gundavelli, K. Leung, V. Devarapalli, K. Chowdhury and B. Patil, Proxy Mobile IPv6, RFC 5213, August 2008.

[9] Ki-Sik Kong and Wonjun Lee, Mobility Management for All-IP Mobile Networks : Mobile IPv6vs, proxy mobile IPv6, IEEE Wireless Communication (April 2008), 36-45.

[10] Jong-Hyouk Lee and Thierry Ernst, Fast PMIPv6 multicast handover procedure for mobility-unaware mobile nodes, Vehicular Technology Conference (VCT Spring) (May 2011), 1-5.

[11] C.E. Perkins, IP Mobility Support, RFC 2002, Oct 1996.

[12] F. Xia and B. Sarikaya, FMIPv6 extension for multicast handover, draft-xiamipshop-fmip-multicast-01, work in progress, March 2007.

[13] J. Guan, H. Luo, H. Zhang, H.-C. Chao and J.H. Park, Design and implementation of light-weight mobile multicast for fast MIPv6, Computer Communications 32 (2009), 552-559.

[14] C. Jelger and T. Noel, Multicast for mobile hosts in IP networks: Progress and challenges, IEEE Wireless Communications 9(5) (2002), 58-64.

[15] I. Romdhani, J. Munoz, H. Bettahar and A. Bouabdallah, Adaptive multicast membership management for mobile multicast receivers, WiMob'2006 (2006), 189-195.

[16] G.A. Leoleis, G.N. Prezerakos and I.S. Venieris, Seamless multicast mobility support using fast MIPv6 extensions, Computer Communications (2006), 3745-3765.

[17] D.H. Kwon, W.J. Kim, Y.S. Kim, W.S. Im and Y.J. Suh, Design and implementation of an efficient multicast support scheme for FMIPv6, INFOCOMM 2006 (2006), 1-12.

[18] T.C. Schmidt and M. Waehlisch, Seamless multicast handover in a hierarchical mobile IPv6 environment (M-HMIPv6), draft-schmidt-waehlisch-mhmipv6-04, work in progress, November 2005.

[19] H.K. Zhang, X.H. Chen and J.F. Guan, Mobile IPv6 multicast with dynamic multicast agent, draft-zhang-mipshopmulticast-dma-03, work in progress, January 2007.

[20] H. Yokota, K. Chowdhury, R. Koodli, B. Patil and F. Xia, Fast Handover for Proxy Mobile IPv6, IETF draft ietf- mipshoppfmipv6-14, May 2010.

[21] J.-H. Lee et al., A novel inter-LMD handoff mechanism for network-based localized mobility management, Lecture Notes in Computer Science (LNCS) 4712 (2007), 356-366.

[22] M. Lebsch et al., Route optimizations for proxy mobile IPv6, IETF, draft-abeillenetlmm-proxymip6ro-01 (Working progress), November 2007.

[23] R. Vida and L. Costa, Multicast listenet discovery version 2 (MLDv2) for IPv6, IETF RFC 3810, June 2004.

[24] T.C. Schmidt, M. Waehlisch and S. Krishnan, Base deployment for multicast listener support in PMIPv6 domains, draftietfmultimob-pmipv6-base-solution-00, February 2010.

[25] B. Fenner, H. He, B. Haberman and H. Sandick, Internet group management protocol/multicast listener discovery based multicast forwarding (IGMP/MLD Proxying), RFC 4605, August 2006.

[26] I. Romdhani, M. Kellil and H.Y. Lach, IP mobile multicast: Challenges and solutions, IEEE Communications 6(1) (2004), $18-41$.

[27] T. Narten, E. Nordmark and W. Simpson, Neighbor discovery for IP version 6 (IPv6), RFC 2461, December 1998.

[28] B.S. Kim and K.J. Han, Multicast handoff agent mechanism for All-IP mobile network, Kluwer Mobile Network and Applications 9 (2004), 185-191.

[29] X.P. Costa, R. Schmitz, H. Hartenstein and M. Liebsch, A MIPv6, FMIPv6 and HMIPv6 handover latency study: Analytical approach, in: Proceedings of IST Mobile \& Wireless Telecommunications Submit (2002).

[30] R. Jain, T. Raleigh, C. Graff and M. Bereschinsky, Mobile Internet Access and QoS Guarantees using Mobile IP and RSVP with Location Registers, ICC'98 (1998), 1690-1695.

Illkyun Im graduated from INHA University in 1992. Now, He is student at Information and Communication Engineering graduate school of SungkyunKwan University, and is working in Process Technology R\&D Group in SAMSUNG SDS Co., 
LTD. His research interests include mobile computing, distributed mobility control, military communication, security, sensor networking, ad hoc networking, M2M, IoT, software and system engineering.

Jongpil Jeong received his B.S. degree in engineering from Sungkyunkwan University and the M.S. and Ph.D. degrees in computer engineering from Sungkyunkwan University, Suwon, Korea, in 2003 and 2008, respectively. He was a Research Professor with Sungkyunkwan University in 2008-2009 and 2011, and a visiting professor with the Department of Interaction Science in Sungkyunkwan University in 2009-2010. He started his academic profession at Sungkyunkwan Univeristy, Korea in 2012 as an assistant professor. His research interests include mobile computing, mobility management for vehicular networks, sensor networking, protocol operation based performance analysis, Internet security, MIPv6 and ubiquitous computing. 

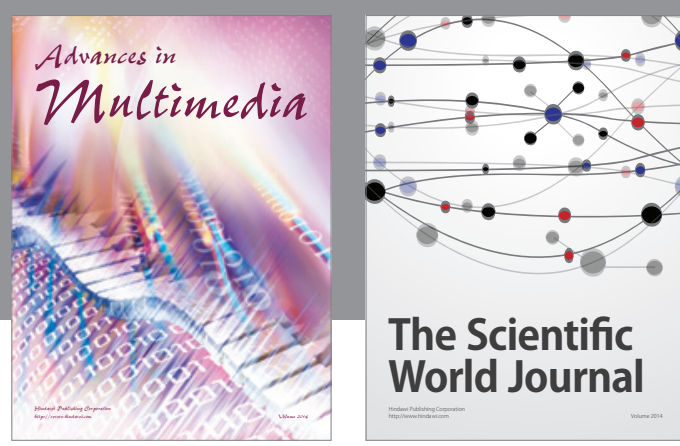

The Scientific World Journal
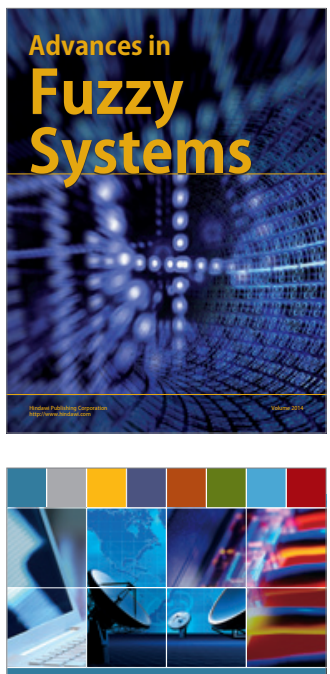

Computer Networks and Communications
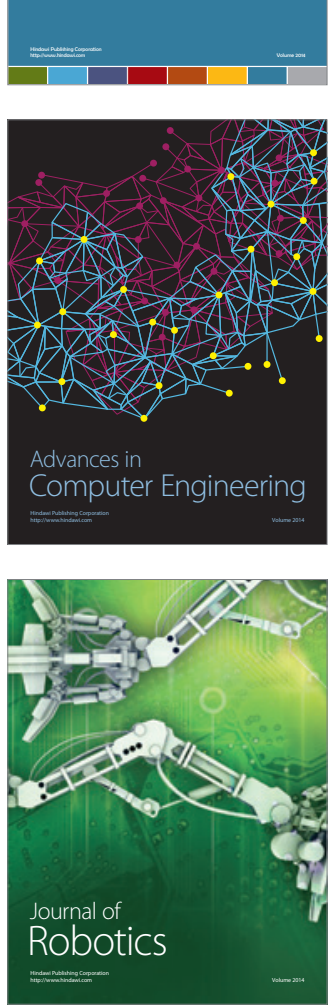
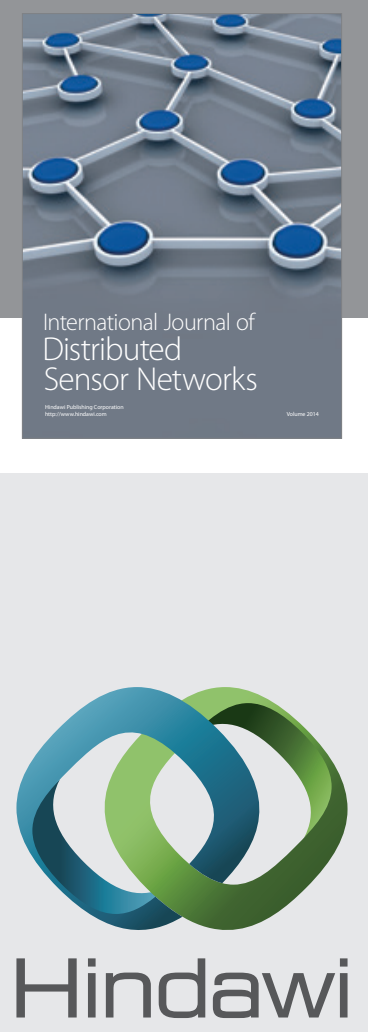

Submit your manuscripts at

http://www.hindawi.com
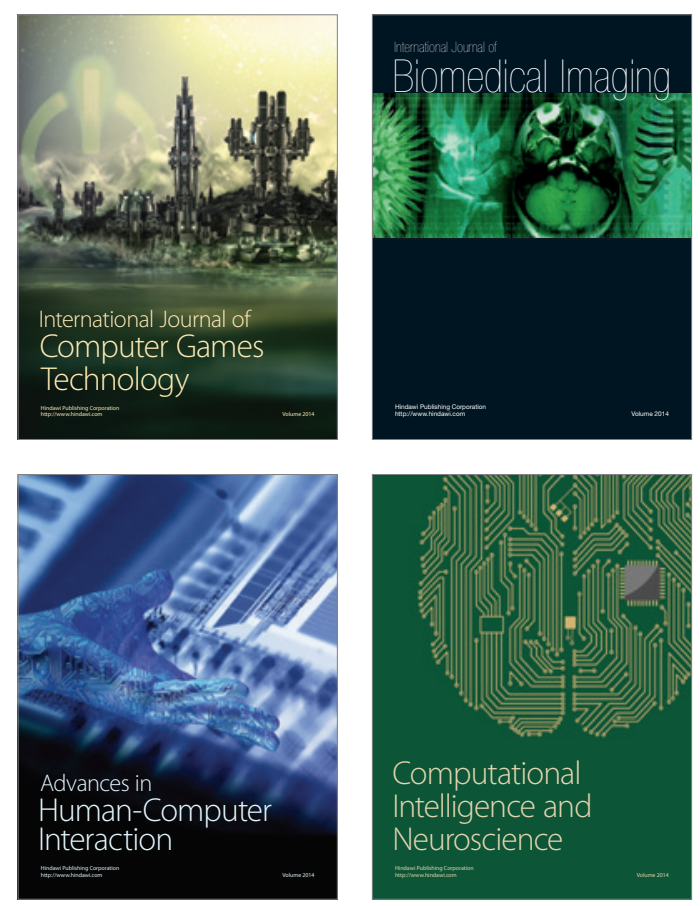
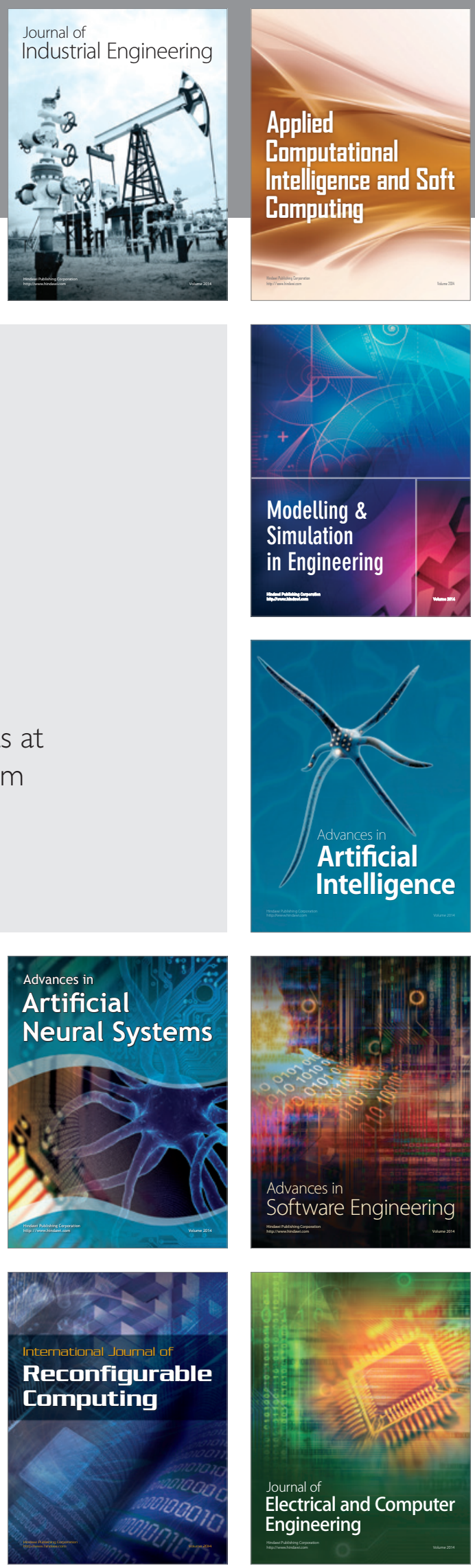